\title{
EL TELETRABAJO Y SU INCIDENCIA EN LA PERCEPCIÓN DE LA FELICIDAD LABORAL
}

\section{TELEWORKING AND ITS IMPACT ON THE PERCEPTION OF JOB HAPPINESS}

\author{
María De Los Ángeles Santana López ${ }^{1}$, Jorge Manuel Cueva Estrada ${ }^{2}$, \\ Nicolás Armando Sumba Nacipucha ${ }^{3}$, Stella Paola Delgado Figueroa ${ }^{4}$
}

\author{
Palabras clave: \\ Teletrabajo, \\ Felicidad Laboral, \\ Desarrollo \\ Organizacional, \\ Clima \\ Organizacional.
}

Resumen

El teletrabajo fue una alternativa para que el mundo continúe sus actividades económicas, durante la pandemia por la COVID-19, su presencia ha mostrado nuevas oportunidades en el mundo empresarial, sin embargo, se hace necesario indagar sobre el bienestar y la felicidad de los empleados que laboran en esta modalidad de trabajo. El objetivo del estudio fue: analizar la incidencia del teletrabajo, sobre la felicidad laboral en los colaboradores de la ciudad de Guayaquil. Para esto se realizó un estudio de tipo descriptivo tanto de variables cualitativas como cuantitativas, las entrevistas se realizaron a cinco expertos en áreas de talento humano y gerentes de empresas, mientras que la encuesta fue dirigida a 387 trabajadores que hayan realizado trabajo presencial y teletrabajo. Se observa un decremento en los niveles de satisfacción laboral a través de la modalidad de teletrabajo. Se concluye que los niveles de felicidad laboral por parte de los trabajadores han sido influenciados negativamente durante el teletrabajo debido a factores como: el aumento de actividades y el hecho de no contar con las herramientas y el ambiente adecuado para ejercer su labor.

Códigos JEL: M12, M54

\footnotetext{
${ }^{1}$ Universidad Politécnica Salesiana, (Ecuador)

E-mail: msantanal@est.ups.edu.ec

${ }^{2}$ Universidad Politécnica Salesiana, (Ecuador)

E-mail: jcueva@ups.edu.ec

${ }^{3}$ Universidad Politécnica Salesiana, (Ecuador)

E-mail: nsumba@ups.edu.ec

${ }^{4}$ Universidad Politécnica Salesiana, (Ecuador)

E-mail: sdelgadof@ups.edu.ec
} 


Keywords:
Telework, Work
Happiness,
Organizational
Development,
Organizational
Climate

\section{INTRODUCCIÓN}

El mundo se ha visto afectado por la pandemia creada por el virus Covid-19, Iturralde y Duque (2021) indican que esta situación obligó a los gobiernos del mundo a decretar un confinamiento obligatorio en toda su población, esto con la finalidad de detener el avance del virus. Este escenario cambió totalmente el ambiente donde las organizaciones realizaban sus actividades (Pérez-Calle, García-Casarejos y García-Bernal, 2021). También exigió a las organizaciones adaptar sus actividades presenciales por unas virtuales, por lo que las jornadas laborales de muchas personas se trasladaron a los hogares de cada uno de ellos, para algunos colaboradores fue su primera experiencia en este ámbito laboral denominado teletrabajo. Rodríguez (2007), describe al teletrabajo como un conjunto de actividades que los colaboradores de las empresas realizan desde su hogar, recibiendo instrucciones y directrices de sus líderes a través de internet, instrucciones que deben cumplirse a cambio de una remuneración mensual, esta actividad implica el no desplazamiento del trabajador hacia las instalaciones de las empresas.

El teletrabajo ha repotenciado actualmente su aplicación e importancia de forma global, esto gracias a la pandemia, donde muchas personas $o$ colaboradores experimentaron por primera vez el trabajo desde un lugar donde no sea la empresa, situación que mostró problemas administrativos, financieros, operativos y humanos que deben ser superados a través de una regulación adecuada de esta actividad (Iturralde y Duque 2021). La fuerza laboral en mucho de los casos tuvo que adaptar un lugar o adaptarse a un espacio dentro de sus viviendas para el desarrollo de sus actividades productivas, ahora las actividades de oficina se desarrollan con la compañía de los niños, con el ruido que producen las mascotas, quizás una mala iluminación, baja velocidad en el internet, clima no adecuado, mala calidad de tecnología y diferentes distractores propios de un hogar (Lampert y Poblete, 2018). Conviene indicar que las actividades a distancia pueden dar paso a situaciones como un horario extendido de trabajo y como consecuencia a un incremento de las responsabilidades laborales (Organización Internacional del Trabajo, 2020). Factores que pudieran haber incidido negativamente en la percepción de felicidad laboral en los trabajadores $\mathrm{y}$ en consecuencia disminuir sus niveles de productividad, en este sentido Bermeo et al. (2019), mencionan que los gerentes de las organizaciones están en la obligación de estudiar los diferentes factores que pudieran incidir positiva o negativamente sobre la productividad de sus empresas. 
Por otra parte, el ambiente de una oficina en la mayoría de los casos ofrece las comodidades necesarias y el trabajador puede concentrarse directamente en su labor, sin distracciones, lo cual le permitiría alcanzar objetivos diarios en su trabajo y en consecuencia aumentar su percepción de felicidad laboral, conviene mencionar que la felicidad laboral es una meta o un fin, para obtener bienestar y permanencia en el trabajo, también motiva el bienestar y el rendimiento de los colaboradores, y en consecuencia cumplir con todos los objetivos y procesos de la empresa (Moccia, 2016).

Desde otro punto de vista, el teletrabajo ha permitido a muchos trabajadores compartir una mayor cantidad de tiempo con sus familiares, situación que en la presencialidad era escasa dado que los miembros del hogar se tenían que desplazar a los diferentes lugares donde desarrollan sus actividades diarias, lo cual minimizaba el tiempo de convivencia con la familia, por parte de los colaboradores (Caamaño,2010).

La pandemia ha permitido que el mundo observe nuevas oportunidades y formas de realizar las cosas que se desarrollan diariamente, ya no es necesario viajar grandes distancias para realizar una reunión de trabajo, ni tampoco es necesario asistir a un puesto de trabajo para realizar una actividad productiva, sin duda el teletrabajo llegó para quedarse en el contexto empresarial, esto dado que supone beneficios para la organización como el ahorro en el consumo de servicios básicos, así como la disminución de espacios físicos de trabajo, por lo que se vuelve necesario para las empresas contar con investigaciones actualizadas acerca del teletrabajo y su incidencia en la felicidad laboral, esto con la finalidad de buscar constantemente una mejora en la productividad de la organización (Buitrago,2020).

Considerando el contexto descrito anteriormente, se pretende responder a la siguiente pregunta de investigación: ¿Qué efectos ha tenido el teletrabajo en la felicidad laboral de los trabajadores de la ciudad de Guayaquil? Por lo tanto, el objetivo que guiará el estudio es: analizar la incidencia del teletrabajo, sobre la felicidad laboral en los colaboradores en la ciudad de Guayaquil. Con los resultados obtenidos los empresarios podrán proponer y ejecutar estrategias para mantener o mejorar los niveles de felicidad laboral y por lo tanto aumentar los niveles de productividad en los colaboradores que realizan sus actividades a través de la modalidad de teletrabajo, factores de mucha importancia sobre todo en tiempos de recesión económica.

\section{Desarrollo organizacional}

Toda empresa busca constantemente alcanzar sus objetivos y en consecuencia el éxito en su segmento de mercado y dado a que las organizaciones son sistemas sociales uno de los elementos fundamentales para alcanzar su desarrollo es el factor humano o su fuerza laboral, quienes deben poseer una actitud competente y alineada a los intereses de la organización, con la finalidad de que aporten constantemente a los fines que persigue la empresa (Segredo, 2015). Muchas organizaciones aún mantienen un pensamiento individualista otorgando el éxito de la empresa a la gestión y administración de un gerente, cuando en realidad la organización es una sociedad de personas que trabajan de forma sistemática para lograr su desarrollo, por lo que la importancia del trabajo en equipo se revalora, con base en lo anterior los individuos que forman parte de la organización deben ser consideradas como el principal activo de las empresas. Se vuelve necesario entonces que la organización cuente con directivos que posean esta visión y personal que aporten constantemente al desarrollo de la organización (Segredo, García, Cabrera, León y Perdomo, 2016). Por lo tanto, las organizaciones deben buscar estrategias que permitan aumentar o mantener buenos niveles de felicidad laboral en sus trabajadores, pues este elemento genera bienestar y permite aumentar el rendimiento de los colaboradores, lo cual ayudará a alcanzar el desarrollo organizacional (Moccia, 2016).

Para Díaz (2019), el desarrollo organizacional es un área del trabajo humano y del conocimiento que se enfoca en la innovación y la transformación de los procesos de las organizaciones empresariales. $\mathrm{Su}$ principal objetivo es favorecer al bienestar de los 
colaboradores de la empresa gracias al acrecentamiento en los niveles de rendimiento de la organización, buscando ayudar al desarrollo social y económico. Con todo lo expuesto se observa que el desarrollo organizacional se presenta cuando los procesos son más eficientes y eficaces, para alcanzar esto es necesario enfocar las luces hacia el talento humano y su felicidad dentro del trabajo.

\section{Cultura organizacional}

La cultura de una empresa engloba un conjunto de pensamientos, hábitos, tradiciones y valores empleados por las personas que forman una empresa $\mathrm{u}$ organización comercial, estas regulan el desempeño de los colaboradores (Pérez y Pérez, 2007). A continuación, se puede resaltar lo expuesto por Soria, (2008) quien afirma que la cultura organizacional se ha transformado en un medio relevante para estimular y desarrollar el rendimiento de las empresas, dado que observa y reconoce las capacidades en el trabajo, el intercambio de ideas y conocimientos entre todas las personas y grupos dentro de la empresa. En este sentido Salazar, Guerrero, Rodríguez y Cañedo, (2009), afirman que el clima o cultura organizacional influye de forma significativa en el desempeño o productividad de la organización comercial.

Esto sucede dado que en un clima organizacional adecuado los valores $y$ comportamientos básicos de convivencia entre personas que sean compartidos crearán un ambiente amigable y deseable para estar, en línea con lo anterior, Marulanda, López y López, (2016) agregan que la cultura organizacional puede ser conceptualizada como un pensamiento colectivo y compartido de valores personales, valores culturales y motivación, por lo que las habilidades y el conocimiento son sustentados por la interacción, el estímulo y el apoyo entre los colaboradores de la organización lo cual crea un ambiente de trabajo amigable, situación que afecta positivamente en el comportamiento y la felicidad de los colaboradores y lo que a su vez se reflejará en la productividad de la empresa (Marulanda, López y Cruz, 2018).
Finalmente cabe indicar que toda empresa desarrolla y crea su cultura organizacional en línea con los valores propuestos por la organización comercial, para esto, los encargados de cada departamento deben participar activamente motivando el cumplimiento de los valores institucionales (Reyes y Moros, 2019). Al contar la empresa con un clima organizacional adecuado los empleados se sentirán más motivados por asistir a desarrollar sus labores diarias siendo este otro factor que influye en los niveles de felicidad laboral del personal.

\section{Calidad de vida laboral}

La calidad de vida laboral se fundamenta en la percepción que tienen los integrantes de una empresa acerca de su nivel de insatisfacción o satisfacción en relación con el lugar donde laboran, percepción que siendo negativa puede afectar a los intereses que persigue la organización empresarial (Baitul, 2012). Esto sugiere que lograr un nivel aceptable de calidad laboral ejercerá un impacto positivo sobre el compromiso del colaborador hacia la empresa donde labora (Surienty, Ramayah, Lo y Tarmizi, 2014). En este sentido Cruz (2018) agrega que las empresas que brindan una mejor calidad de vida laboral poseen un mayor poder sobre la retención y contratación del talento humano, elemento valioso que aporta con conocimiento propio para el desarrollo de la organización.

Es importante agregar que una empresa que no valora a su personal y no se preocupa de su calidad de vida laboral puede tener como consecuencia altos niveles de desmotivación, ausentismo, constante rotación del talento humano, así como abandono de los lugares de trabajo (Granados, 2011). La competitividad de las empresas ha aumentado, son más eficientes y sus procesos poseen alta calidad, en todas ellas el talento humano posee un rol relevante, por lo tanto, la calidad de vida laboral toma un papel protagónico en el éxito de la gestión actual.

Finalmente, Bustamante, Álvarez, Villalobos y Lucero (2020) agregan que los factores como la recreación, momentos familiares, reconocimiento de la labor por parte de la empresa, ambiente adecuado de trabajo, integración y apoyo entre 
compañeros influyen en la percepción de calidad de vida laboral.

\section{Felicidad en el trabajo}

La felicidad tiene una significativa ponderación sobre el colaborador, en cualquier tiempo o lugar, si el individuo no es feliz su vida se convierte en rutinaria, sin motivaciones para progresar o alcanzar metas, este tema también se relaciona con la calidad de vida laboral. No existe una sola definición de felicidad pues, esta dependerá del entorno, del tiempo, de la persona y sus anhelos (Margot, 2007; Tierno, 2009), considerando lo expuesto se puede decir que la felicidad laboral se basa tanto en la persona y su voluntad por ser feliz, por otro lado, está el ambiente laboral que ofrece la empresa. En este sentido Malluk (2018) menciona que la felicidad laboral implica la mezcla de la correcta gestión y administración de las operaciones junto con la administración del talento humano para llegar a la felicidad profesional. Esto último hace prever que, si en la felicidad del colaborador de la empresa solo se ponderaría la actitud del individuo, se pudieran realizar pocas actividades para poder incidir sobre ella, sin embargo, al incluir el ambiente laboral este puede ser manipulado a favor de aumentar los niveles de felicidad en los trabajadores (Ramírez-García, García-Álvarez de Perea y García del Junco, 2019).

La felicidad laboral para Vázquez (2012) es una nueva forma de gestión que ofrece la posibilidad de motivar la felicidad de los colaboradores de la empresa mediante los siguientes factores: confianza, optimismo, compromiso y aprendizaje. En esta línea Garzón (2017) señala que la felicidad laboral es el nivel de afecto y de compromiso que posee el empleado hacia su trabajo y agrega que los colaboradores comprometidos generalmente se esfuerzan más de lo normal por ayudar al progreso y desarrollo de la empresa.

Para Ravina-Ripoll, Marchena y MontañésDel Río (2019) la felicidad laboral es el engranaje principal para la gestión de empresas que estimulen el desarrollo de un ambiente agradable $\mathrm{y}$ positivo en su interior, con la finalidad de impulsar la creatividad e innovación en sus empleados. Por otra parte, conviene mencionar lo expuesto por Garzon, Orozco y Ramírez (2020) quienes indican que las variables que integran la felicidad laboral son:

Bienestar subjetivo: Se puede describir como la evaluación que realiza el individuo hacia su propia vida, esta evaluación incluye una observación a áreas como: el matrimonio, el trabajo, la edad, la salud, el estado civil (Díaz, 2001).

Satisfacción laboral: Está claro que un colaborador que no demuestra conformidad hacia las labores que realiza presenta sentimientos negativos que pueden afectar a las actividades empresariales y a su satisfacción laboral, por lo contrario, un colaborador con sentimientos de compromiso y aprecio hacia su labor crea un entorno y una percepción positiva de satisfacción laboral (Díaz, Cárdenas y Martínez, 2012).

Compromiso organizacional: Nivel de implicación o de compromiso del empleado hacia la empresa, el colaborador acepta los valores de la organización, con altos niveles de compromiso por alcanzar los propósitos institucionales, así como el deseo de mantenerse trabajando en ella (Garzón, 2017).

Implicación laboral: Las actividades diarias que desempeña un individuo como su trabajo son un componente de importancia en su vida. Esto sugiere que una persona con implicación laboral en niveles altos es una persona satisfecha y entregada a su labor (Garzón, 2017).

Compromiso individual: Actitud de entrega hacia el trabajo que desarrolla en su entorno laboral, todo el esfuerzo que una persona realiza para cumplir con sus actividades de trabajo (Garzón, 2017).

Prosperidad: Crecimiento en el trabajo mediante la adquisición de conocimientos nuevos y desarrollo profesional (Garzón, 2017).

Afecto al trabajo: Placer y satisfacción personal por el trabajo desarrollado (Garzón, 2017). 


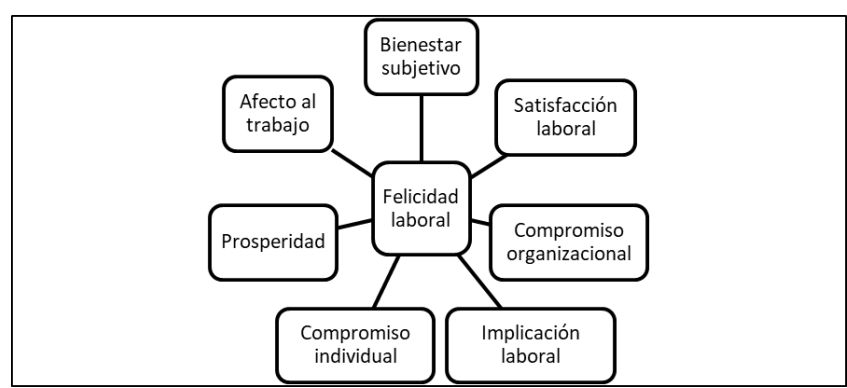

Figura 1. Variables de la Felicidad Laboral.

Fuente: elaboración propia con base en Díaz (2001), Díaz, Cárdenas y Martínez (2012), Garzón (2017)

Considerando lo anterior se puede notar la felicidad laboral es un campo que puede ser estudiado o analizado desde diferentes aristas. Todas las empresas están constituidas por seres humanos quienes llevan a la empresa al éxito, a su desaparición o un estancamiento, sin embargo, bajo el análisis y aplicación de estrategias para controlar las variables antes descritas y que se pueden observar en la figura 1, se pudiera concebir que los colaboradores sean más felices y en consecuencias más productivos.

\section{Teletrabajo}

Se puede describir al teletrabajo como una manera de flexibilizar el trabajo, este se fundamenta en el desarrollo de actividades laborales sin la presencialidad del trabajador en la empresa, requiriendo de tecnologías de la información y comunicación TIC para su correcto desarrollo (Salazar, 2007). Dicho en otras palabras, el teletrabajo son las responsabilidades del trabajo diario, pero ahora desarrollado mediante la ayuda y pilar fundamental de las TIC, esto realizado en un ambiente externo al de las instalaciones de la empresa (Eurofound y OIT, 2019).

Por otra parte, se debe recalcar que el teletrabajo se presenta mediante un pacto voluntario entre la empresa y el colaborador, para esto se debe acordar el lugar donde se desarrollarán las actividades (hogar del trabajador o en cualquier otro lugar), también se debe aclarar las horas dedicadas al teletrabajo y medios de comunicación a utilizarse, cabe indicar que las actividades deberán desarrollarse con métodos de supervisión y control (Organización Internacional del Trabajo, 2020).

En este sentido, estudios realizados acerca de las horas de trabajo de los teletrabajadores desde su hogar- por causa de la presencia del virus COVID19-, informa que el $38 \%$ de los encuestados señalaron que es muy probable que trabajen más horas desde su hogar (McCulley, 2020). En otra investigación, el $27 \%$ afirman que usan su tiempo libre para cubrir el exceso de actividades (Eurofound, 2020). Finalmente, las personas pasan más tiempo en reuniones de trabajo como consecuencia del distanciamiento social (Reisenwitz, 2020).

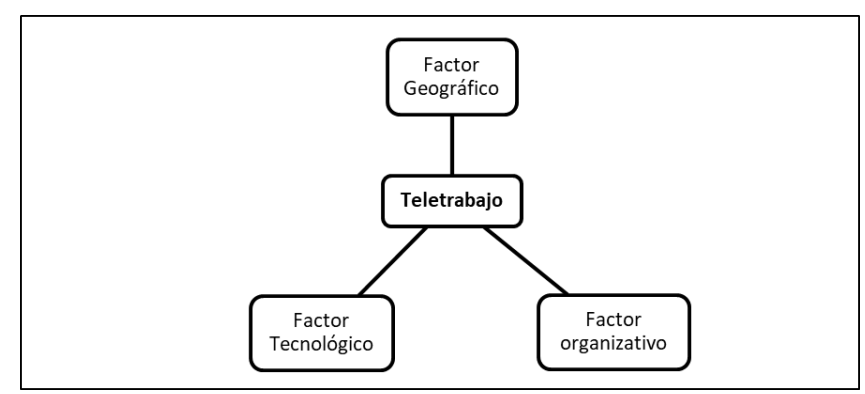

Figura 2. Factores diferenciales del Teletrabajo Fuente: elaboración propia con base en Caamaño, (2010)

El teletrabajo consta de tres factores diferenciales que se pueden observar en la figura 2 y se describen a continuación: Factor geográfico lo cual sugiere que el teletrabajo es una actividad desarrollada a distancia, es necesario comprender que el teletrabajo no solo puede ser desarrollado desde el hogar del colaborador, sino que implica también dar servicio a la empresa desde cualquier lugar fuera de esta. Factor tecnológico esto implica que al colaborador se le debe de suministrar o este debe contar con la tecnología tanto de hardware, software y telecomunicaciones necesarias que faciliten el desarrollo de su actividad fuera de la organización comercial. Conviene indicar que el uso de tecnología en el teletrabajo es intensa pues de ella dependen el éxito de la actividad. Factor organizativo el teletrabajo no implica solo el hecho de trabajar a distancia o fuera de la empresa utilizando medios tecnológicos, sino que debe comprenderse que es una manera de organización y de desarrollo de trabajo de forma descentralizada. Por lo tanto, 
debe ayudar a alcanzar los objetivos empresariales (Caamaño,2010).

Considerando que el teletrabajo guarda las mismas responsabilidades que un trabajo de forma presencial y que el individuo es uno de los pilares principales para alcanzar el éxito en las organizaciones, se vuelve necesario estudiar los niveles de percepción de felicidad laboral en los empleados que realizan teletrabajo.

\section{METODOLOGÍA}

El presente estudio es empírico, transversal y con un enfoque descriptivo tanto cualitativo como cuantitativo. El objetivo principal que guio el estudio fue: analizar la incidencia del teletrabajo, sobre la felicidad laboral en los trabajadores de la ciudad de Guayaquil.

En primer lugar y para alcanzar el objetivo específico 1: Analizar la opinión de expertos acerca del teletrabajo y la felicidad laboral, se realizó un cuestionario con cinco preguntas abiertas, las cuales fueron dirigidas a expertos en el área de gestión del talento humano, así como profesores y gerentes $\mathrm{o}$ administradores de empresas comerciales.

A continuación, para lograr el objetivo específico 2: Reunir la opinión y percepción de trabajadores administrativos acerca del teletrabajo y la felicidad laboral, se realizó un cuestionario formado por 20 preguntas con opciones de respuesta en escala de Likert, el cual fue dirigido a empleados de empresas que hayan experimentado actividades de teletrabajo y trabajo presencial, a fin de poder establecer un contraste entre estas dos modalidades de trabajo. Por los efectos de la pandemia a nivel global las herramientas de investigación mencionadas anteriormente fueron aplicadas mediante el uso de internet y medios digitales. Tanto el cuestionario de la encuesta como el de la entrevista fueron validados por expertos previo a su aplicación.

Para finalizar y lograr el objetivo específico sobre describir el efecto que han tenido las actividades en el teletrabajo sobre la felicidad laboral. Para esto se analizaron los datos obtenidos mediante las herramientas de investigación, Generar gráficos estadísticos que permitan comparar los niveles de felicidad laboral durante el trabajo presencial y el teletrabajo.

El tamaño de la muestra utilizada para la investigación se determinó con información del sitio web del Instituto Nacional de estadísticas y Censo INEC 2021. En su contador poblacional se puede observar un total de 17.697.531 ecuatorianos y tomando como referencia que a nivel nacional el porcentaje de empleo adecuado fue del $31.3 \%$ se tiene una población de interés para la presente investigación de: 5.539 .327 ecuatorianos con empleo adecuado (INEC, 2021). Población que según Triola (2009) debe ser considerada como infinita para el cálculo de la muestra por superar los 100.000 elementos, para la determinación del tamaño muestral se usará una confianza del $95 \%$ y un error máximo del $5 \%$.

$$
n=\frac{z^{2} \pi(1-\pi)}{e^{2}} \quad n=\frac{1.96^{2} 0.5(1-0.5)}{0.05^{2}}=385
$$

\section{RESULTADOS}

\section{Entrevistas}

La opinión de los entrevistados respecto a la mantención y generación de colaboradores felices dentro de las empresas, los entrevistados propusieron: estudiar la cargar de labores de cada una de las personas que integran la organización de tal forma que las responsabilidades sean equitativas, esta situación agrega que incide en un buen ambiente laboral. También mencionaron la creación de un programa de incentivos a los colaboradores, esto por el cumplimiento de metas u objetivos, fomentar la sana competencia entre los colaboradores de la organización. Adicionalmente agregaron y resaltaron la importancia de empoderar a las personas en su trabajo de tal forma que estos tengan cierta libertad para tomar decisiones y sentir que su trabajo es importante y ayuda al cumplimiento de los objetivos que la empresa persigue.

En lo concerniente a la felicidad del colaborador desde el teletrabajo los entrevistados concuerdan que este ha causado una mayor carga de actividades e incluso mencionan que se ha perdido el respeto en el uso de las horas libres del trabajador, sin embargo, también mencionan que 
los colaboradores pueden realizar sus labores personales durante el día y concluir con su carga laboral por la noche, esta situación indica que puede crear una falsa percepción de sobrecarga laboral.

"Tener colaboradores felices aumenta la productividad de las organizaciones" los entrevistados comparten totalmente esta afirmación, mencionan que los trabajadores felices están más predispuestos al trabajo, situación que se ve reflejada en una correcta atención al cliente externo e interno, así como en una adecuada adaptación en momentos en que el trabajo amerite la extensión de la jornada. Por otra parte, añaden que la felicidad laboral no solo depende del trabajador sino también en gran medida de la habilidad que tenga la empresa por generar un adecuado ambiente de trabajo. Para finalizar y al respecto de cómo medir la felicidad laboral, los expertos señalan que las encuestas es una herramienta válida pues recoge la percepción del colaborador, sin embargo recomienda aplicar herramientas como la observación y el constante diálogo con los colaboradores pues estas últimas permite recoger resultados en ambientes $y$ situaciones reales.

\section{Encuestas}

La encuesta fue contestada por trabajadores del área administrativa quienes pertenecían a una empresa y trabajaban de manera presencial y que luego por efectos de la pandemia realizaron teletrabajo, a continuación, se compara los resultados de la encuesta realizada referente a la felicidad laboral durante el trabajo presencial y el teletrabajo.

Nomenclatura utilizada en los gráficos, Totalmente en desacuerdo (TDES), En desacuerdo (DES), $\mathrm{Ni}$ en desacuerdo ni de acuerdo (NDES/NA), De acuerdo (DA), Totalmente de acuerdo (TD). En lo referente a la variable BIENESTAR SUBJETIVO (BS), la figura 3 muestra un descenso en la satisfacción que provocaba la situación en su entorno familiar después del inicio de la pandemia, esto puede deberse a que muchos hogares tuvieron muertes de familiares en las épocas más duras de la crisis de salud.

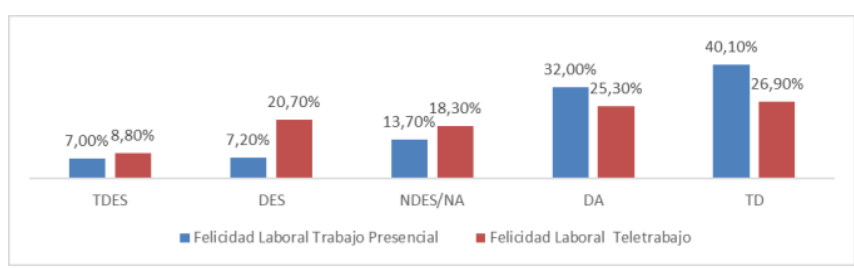

Figura 3. (BS) Mi situación familiar me provocaba felicidad

En esta misma variable, pero en lo referente al estado de salud para trabajar, la figura 4 permite observar un decremento en la percepción por parte de los encuestados acerca de su buen estado de salud.

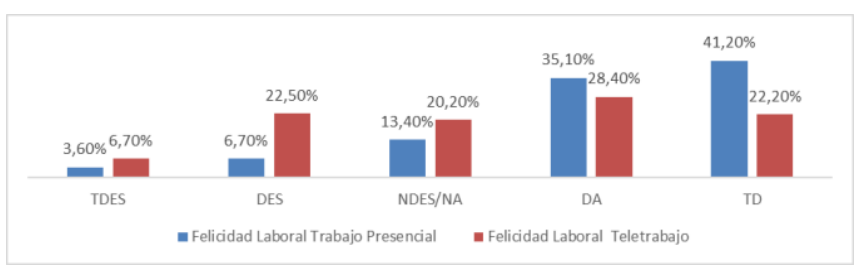

Figura 4. (BS) Mi salud era la adecuada para trabajar

Los resultados obtenidos en la variable bienestar subjetivo muestran valores negativos que inciden en la felicidad laboral de los trabajadores.

Acerca de la variable SATISFACCIÓN LABORAL (SL), las figuras 5, 6 y 7 dejan observar una disminución en los niveles de acuerdo y un aumento en los niveles en desacuerdo durante el teletrabajo que en el trabajo presencial.

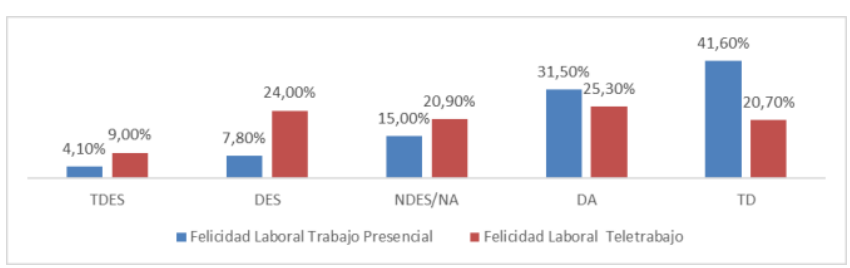

Figura 5. (SL) Siento mucha estima y aprecio por el trabajo que realizo en la empresa 


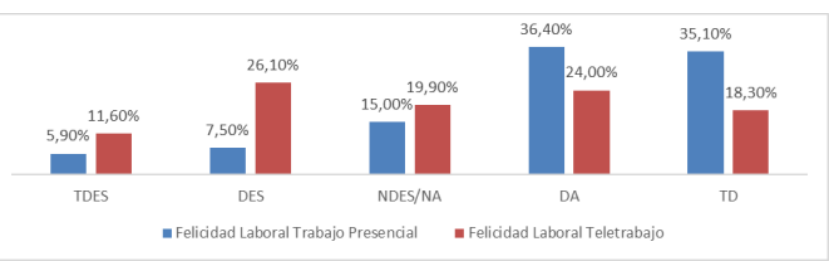

Figura 6. (SL) Cuento con todo lo necesario para desarrollar mis labores

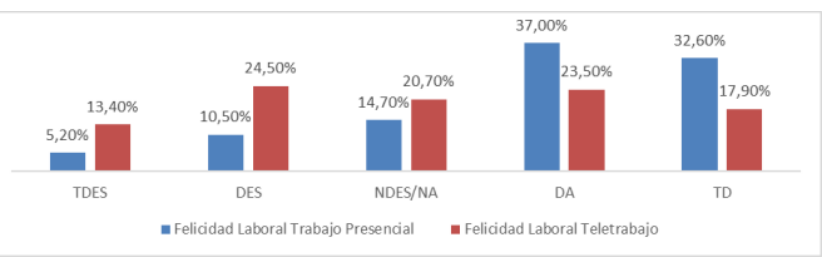

Figura 7. (SL) El ambiente donde trabajo es el adecuado

Acerca de las siguientes variables de la felicidad laboral Compromiso organizacional (CO), Implicación laboral (IL), Compromiso individual (CI), Prosperidad (PR), Afecto en el trabajo (AT), las figuras 8, 9, 10, 11, 12 confirman la tendencia en la disminución de los niveles de satisfacción por parte de los encuestados.

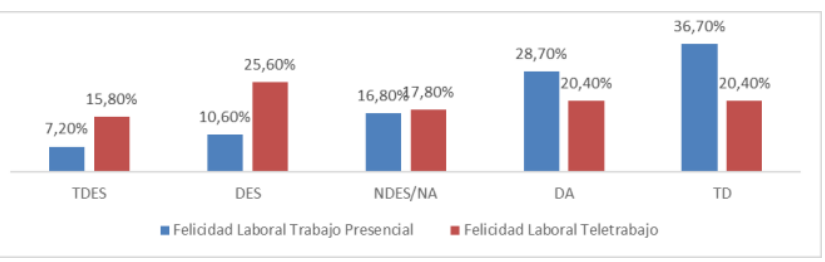

Figura 8. (CO) Deseo continuar ejerciendo mi trabajo en la empresa donde laboro

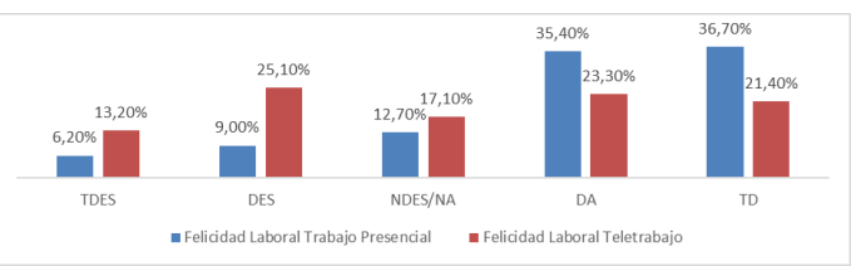

Figura 9. (IL) Realizo las actividades de mi trabajo porque me gustan y siento que son importantes

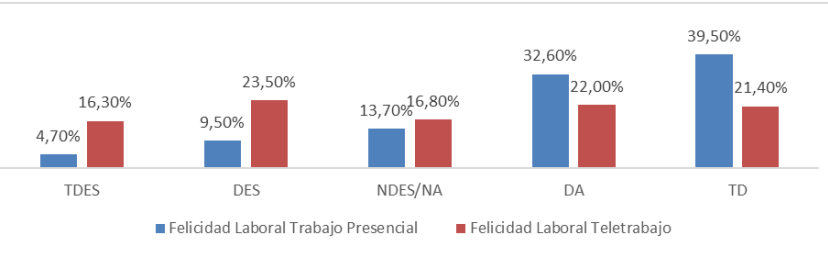

Figura 10. (CI) Me siento comprometido por cumplir con las metas y objetivos de mi puesto de trabajo

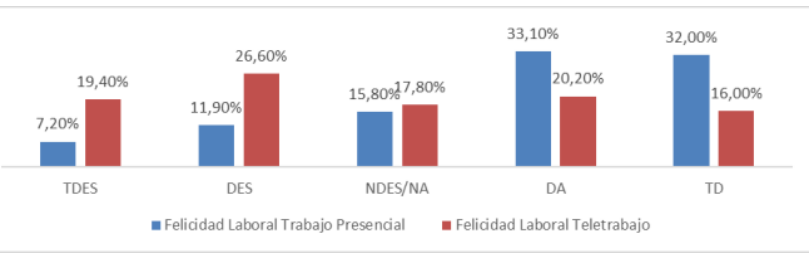

Figura 11. (PR) Mi trabajo me otorga premios o incentivos por el trabajo o conocimiento adquirido

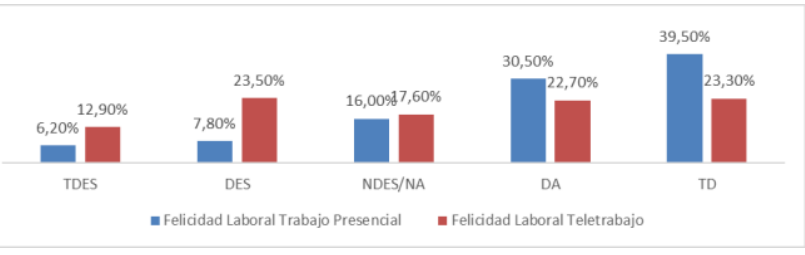

Figura 12. (AT) Disfruto haciendo mi trabajo

Los resultados obtenidos en cada uno de los 10 pares de preguntas demuestran la descendencia en los niveles de acuerdo y totalmente de acuerdo, mientras que también permiten observar el aumento en los niveles en desacuerdo $\mathrm{y}$ totalmente en desacuerdo, comparando los niveles de felicidad laboral durante el trabajo presencial y durante el teletrabajo.

\section{CONCLUSIONES}

Las organizaciones comerciales están en permanente búsqueda del aumento de su productividad y en consecuencia incrementar sus ganancias, En este sentido la revisión de literatura permitió observar que para que estos logros sean alcanzado por las empresas, éstas deben trabajar en un pilar fundamental: las personas que integran a la organización, aumentar sus niveles de bienestar, así como mejorar el clima organizacional incidirá en los niveles de felicidad laboral, lo que repercutirá positivamente en la productividad de la organización, pues el hecho 
de tener empleados felices influye positivamente en todo el trabajo que se realiza dentro de la empresa.

Los resultados obtenidos a través de la encuesta hacen prever que los niveles de felicidad laboral en el teletrabajo han disminuido si se comparan con los niveles de felicidad laboral durante las actividades presenciales, esto puede deberse según los resultados a que dentro del hogar no existen las condiciones necesarias para que el empleado pueda realizar sus actividades, pues existen distractores e incluso un clima no adecuado para ejercer el trabajo, lo cual no permite que la persona pueda alcanzar objetivos de trabajo diarios. También se puede atribuir al aumento en la asignación de actividades por parte de la empresa hacia el colaborador situación que aumenta el nivel de estrés y cansancio en los trabajadores.

Las investigaciones revisadas en el presente estudio concluyen que la felicidad laboral no es solo un bienestar subjetivo, sino que también es un conjunto de variables que pueden ser trabajadas por parte de la empresa para aumentar la satisfacción y el compromiso de los trabajadores hacia la empresa.

El teletrabajo incursiono bruscamente en el entorno empresarial y a nivel global, esto debido a la presencia nefasta de la enfermedad COVID19, esta modalidad de trabajo sin lugar a dudas ha llegado para quedarse, se vuelve necesario entonces que las actividades de teletrabajo sean reguladas con normativas claras y precisas, que defiendan el trabajo y los intereses del colaborador. Así mismo estas normativas deben garantizar la continuidad de las actividades empresariales y el cumplimiento de éstas por parte de sus trabajadores.

\section{REFERENCIAS}

Bermeo, Pérez, Gómez, Borda, Valencia, Ávila y Tamayo (2019) influencia de la felicidad en la productividad de los trabajadores en las organizaciones de Colombia DOI:10.13140/RG.2.2.12359.65447
Baitul, M. (2012). Factors affecting quality of work life: An. Global Journal Of Managemente And Business Research, 12(18).

Buitrago Botero, Diego Martín. (2020). Teletrabajo: una oportunidad en tiempos de crisis. Revista CES Derecho, 11(1), 1-2. https://bit.ly/3zifZPB

Bustamante, Miguel A., Álvarez, Alejandra J., Villalobos, Macarena E., \& Lucero, Miguel I.. (2020). Percepción de la calidad de vida laboral de los trabajadores de los centros de salud familiar de la zona central de Chile. Información tecnológica, 31(3), 65-74. https://dx.doi.org/10.4067/S071807642020000300065

Caamaño Rojo, Eduardo. (2010). El teletrabajo como una alternativa para promover y facilitar la conciliación de responsabilidades laborales y familiares. Revista de derecho (Valparaíso), (35), 79-105. http://dx.doi.org/10.4067/S071868512010000200003

Cruz Velazco, J. E. (2018). La calidad de vida laboral y el estudio del recurso humano: una reflexión sobre su relación con las variables organizacionales. Revista científica Pensamiento Y Gestión, (45). https://rcientificas.uninorte.edu.co/index.php/pen samiento/article/view/10617

Díaz Llanes, Guillermo. (2001). El bienestar subjetivo: Actualidad y perspectivas. Revista Cubana de Medicina General Integral, 17(6), 572579.

http://scielo.sld.cu/scielo.php?script=sci_arttext \&pid=S0864-

$21252001000600011 \& \operatorname{lng}=\mathrm{es} \& \operatorname{tn} \mathrm{ln}=\mathrm{es}$.

Díaz, M. G. D., Cárdenas, M. C. P. \& Martínez, A. K. G. (2012). La comunicación y el grado de satisfacción en las relaciones interpersonales de los trabajadores de una pequeña empresa de la industria metal mecánica. In Global Conference on Business \& Finance Proceedings (Vol. 7, No. 2, p. 959). Institute for Business \& Finance Research. GCBF Vol. 7 No. 2 2012, ISSN 19419589 ONLINE \& ISSN 1931-0285 CD 
Díaz Pérez, Maiky. (2019). Preliminary Study about Organizational Development and Planed Change in an Organization of the Services Sector. Universidad de La Habana, (288), 245-260. Epub 01 de septiembre de 2019, de http://scielo.sld.cu/scielo.php?script=sci_arttext \&pid=S0253-

$92762019000200245 \& \operatorname{lng}=e s \& t \operatorname{lng}=e n$.

Eurofound y OIT. (2019). Trabajar en cualquier momento y en cualquier lugar: consecuencias en el ámbito laboral. Ginebra: OIT. https://www.ilo.org/wcmsp5/groups/public/--ed_protect/---protrav/---

travail/documents/publication/wcms_712531.pdf

Eurofound. (2020). Vida, trabajo y COVID-19 Primeras conclusiones - Abril de 2020. Dublin: Eurofound.

https://www.eurofound.europa.eu/es/publications /report/2020/\%20living-working-and-covid-19first-findings-april-2020

Feliz Marrero, J. (2020). Efectos del Covid-19 en los mecanismos de coordinación e integración de la arquitectura organizacional en las empresas. Ciencia, Economía y Negocios, 4(2), 107-129. Doi:

https://doi.org/10.22206/ceyn.2020.v4i2.pp107129

Garzón-Castrillon M. (2017) Cómo gestionar la felicidad en el trabajo Revista Ide@s Concyteg, 12 No 161, pp,1-21. https://bit.ly/37VfezT

Garzon Castrillon, M. A., Orozco Quintero, D., \& Ramírez Gañan, A. E. (2020). Management of happiness, subjective well-being and job satisfaction. Dimensión Empresarial, 18(2). https://doi.org/10.15665/dem.v18i2.2057

Granados, I. (2011). Calidad de vida laboral: historia, dimensiones y beneficios. Revista IIPSI, 14(2), 13. http://pepsic.bvsalud.org/pdf/ripsi/v14n2/a14.pdf

Iturralde Durán, C. A., \& Duque Romero, L. E. (2021). PRECARIZACIÓN DEL TELETRABAJO EN ECUADOR EN CONTEXTO DE COVID-19: VARIABLES DE ANÁLISIS DESDE EL ENFOQUE
MARXISTA. Chakiñan, Revista De Ciencias Sociales Y Humanidades, (14), 146-162. https://doi.org/10.37135/chk.002.14.10

Instituto Nacional de Estadísticas y Censo INEC. (2021). Encuesta Nacional de

Empleo, Desempleo y Subempleo (ENEMDU). https://www.ecuadorencifras.gob.ec/documentos /web-inec/EMPLEO/2021/Junio-

2021/Boletin_empleo_220721_DEAN3\%20(1).p df

Lampert, M., Poblete, M. (2018,10,24). Efectos positivos y negativos del teletrabajo sobre la salud. Biblioteca del Congreso Nacional de Chile. Recuperado de https://bit.ly/3jdslSj

Margot, Jean-Paul. (2007). LA FELICIDAD. Praxis Filosófica, (25), 55-80. http://www.scielo.org.co/scielo.php?script=sci_a rttext\&pid=S0120-

$46882007000200004 \& \operatorname{lng}=\mathrm{en} \& \operatorname{tlng}=\mathrm{e}$

Marulanda, Carlos, López, Marcelo, \& López, Fernando. (2016). La Cultura Organizacional y las Competencias para la Gestión del Conocimiento en las Pequeñas y Medianas Empresas (PYMEs) de Colombia. Información tecnológica, 27(6), 03-10. https://dx.doi.org/10.4067/S0718-

07642016000600002

Marulanda, Carlos, López, Luis, \& Cruz, Gabriel. (2018). La Cultura Organizacional, Factor Clave para la Transferencia de Conocimiento en los Centros de Investigación del Triángulo del Café de Colombia. Información tecnológica, 29(6), 245-252.

https://dx.doi.org/10.4067/S071807642018000600245

Malluk, Ana. (2018). Felicidad organizacional: mediación entre la valoración del empleado y la productividad. Una mirada a su gestión en el sector estatal. Anagramas Rumbos y Sentidos de la Comunicación, Vol. 17 Núm. 33,. 215-250. http://www.scielo.org.co/pdf/angr/v17n33/16922522-angr-17-33-215.pdf 
Minsal Pérez, Delaray, \& Pérez Rodríguez, Yudit. (2007). Hacia una nueva cultura organizacional: la cultura del conocimiento. ACIMED, 16(3), de http://scielo.sld.cu/scielo.php?script=sci_arttext \&pid=S1024-

$94352007000900008 \& \operatorname{lng}=\mathrm{es} \& \operatorname{tn} g=\mathrm{es}$.

Moccia, Salvatore (2016). FELICIDAD EN EL TRABAJO. Papeles del Psicólogo, 37(2),143-151 https://www.redalyc.org/articulo.oa?id=7784605 5007

McCulley, L. 2020. Lockdown: Homeworkers putting in extra hours - instant messaging up 1900\%. 27 de abril. Disponible en https://www.thehrdirector.com/businessnews/theworkplace/new-data-over-a-third-38admit-to-working-longer-hours-when-workingfromhome/

Organización Internacional del Trabajo. (2020). El teletrabajo durante la pandemia de COVID-19 y después de ella. https://www.ilo.org/wcmsp5/groups/public/--ed_protect/---protrav/---

travail/documents/publication/wcms_758007.pdf

Pérez-Calle, R.D., García-Casarejos, N., y García-Bernal, J. (2021). La empresa española ante la COVID-19: factores de adaptación al nuevo escenario. Retos Revista de Ciencias de la Administración y Economía, 11(21), pp. 5-24. https://doi.org/10.17163/ret.n21.2021.01

Ramírez García, C., García Álvarez de Perea, J. y García del Junco, J. (2019). La felicidad en el trabajo: validación de una escala de medida. RAE: Revista de Administración de Empresas, 59 (5), 327-340.

https://www.scielo.br/j/rae/a/9RRDJ3T5wDwnP D8xLwp6FLK/?lang=es

Ravina-Ripoll, R., Marchena Domínguez, J., y Montañés-Del Río, M.A. (2019). Happiness Management en la época de la Industria 4.0. Retos Revista de Ciencias de la Administración y Economía, $9(18)$, 189-202. https://doi.org/10.17163/ret.n18.2019.01.
Reyes Hernández, Javier, \& Moros Fernández, Helena. (2019). La cultura organizacional: principales desafíos teóricos y metodológicos para su estudio. Revista Estudios del Desarrollo Social: Cuba y América Latina , 7(1), 201-217. Epub 01 de septiembre de 2019, de http://scielo.sld.cu/scielo.php?script=sci_arttext \&pid=S2308

01322019000100201\&lng=es\&tlng=es

Reisenwitz, C. (2020). How COVID-19 is impacting workers' calendars. Clockwise blog, 21 de abril. https://www.getclockwise.com/blog/how-covid19-is-impacting-workerscalendars

Rodríguez Mejía, Marcela. (2007). El teletrabajo en el mundo y Colombia. Gaceta Laboral , 13(1), $29-42$.

http://ve.scielo.org/scielo.php?script=sci_arttext \&pid=S1315$85972007000100002 \& \operatorname{lng}=$ es\&tlng=es.

Salazar-Concha, C. (2007). El Teletrabajo Como Aporte a la Inserción Laboral de Personas con Discapacidad en Chile: Una Gran Carretera Virtual por Recorrer. Ciencia \& Trabajo. 9 (25). 89-98. https://bit.ly/3gk46ky

Segredo Pérez, A., García Milian, A., León Cabrera, P., \& Perdomo Victoria, I. (2016). Desarrollo organizacional, cultura organizacional y clima organizacional. Una aproximación conceptual. Revista De Información Científica Para La Dirección En Salud. INFODIR, 0(24), 8699. do de http://revinfodir.sld.cu/index.php/infodir/article/ view/200

Segredo Pérez, Alina María. (2015). Desarrollo organizacional. Una mirada desde el ámbito académico Escuela Nacional de Salud Pública, La Habana, Cuba http://dx.doi.org/10.1016/j.edumed.2015.09.002

Soria, R. (2008). Emprendurismo, cultura, clima y comunicación organizacional y su aplicación a la pequeña y mediana empresa en la Zona Metropolitana de Guadalajara, México. España: Red Académica Iberoamericana Local-Global, Grupo EUMEDNET Universidad de Málaga. 
https://es.scribd.com/document/359543859/Empr endurismo-Cultura-Clima-y-Comunicacion-

Organizacional-y-Su-Aplicacion-a-La-Pequenay-Mediana-Empresa

Surienty, L., Ramayah, T., Lo, M. C. y Tarmizi, A. N. (2014). Quality of work life and turnover intention: a partial least square (PLS) approach. Social Indicators Research, 119(1), 405-420. doi: https://doi.org/10.1007/s11205013-0486-5

Salazar Estrada, José Guadalupe, Guerrero Pupo, Julio Cristóbal, Machado Rodríguez, Yadira Bárbara, \& Cañedo Andalia, Rubén. (2009). Climate and organizational culture: two essential components in the working productivity. ACIMED, 20(4), 67-75, de http://scielo.sld.cu/scielo.php?script=sci_arttext \&pid=S1024-

$94352009001000004 \& \operatorname{lng}=\mathrm{es} \& \ln \mathrm{l}=\mathrm{en}$

Tierno, B. (2009). Los pilares de la felicidad. Vivir mejor, Editor Booket: Madrid.

Triola, F. (2009). Estadística. Décima edición, México: Pearson Educación. ISBN: 978-970-261287-2.

Vázquez, S. (2012). La felicidad en el trabajo y en la vida. Madrid, España: Actualia Editorial 